\title{
ESTIMULAÇÃO CALÓRICA GELADA COM AR NAS VESTIBULOPATIAS PERIFÉRICAS CRÔNICAS COM NISTAGMO ESPONTÂNEO
}

\author{
Ice air caloric test in chronic peripheral vestibular dysfunction \\ with spontaneous nystagmus
}

\author{
Flavia Silveira dos Santos Cabral (1), Cristina Freitas Ganança ${ }^{(2)}$, Fernando Freitas Ganança ${ }^{(3)}$, \\ Maurício Malavasi Ganança ${ }^{(4)}$, Heloisa Helena Caovilla ${ }^{(5)}$
}

\section{RESUMO}

Objetivo: analisar o efeito da estimulação gelada com ar a $10^{\circ} \mathrm{C}$ sobre o nistagmo pós-calórico em pacientes com vestibulopatias periféricas crônicas que apresentam nistagmo espontâneo com olhos fechados. Métodos: 61 pacientes foram submetidos às estimulações com ar a 42,18 e $10^{\circ} \mathrm{C}$. Resultados: em 42 casos (69,8\%) foram encontrados valores anormais de preponderância direcional e/ou de predomínio labiríntico a 42 e $18^{\circ} \mathrm{C}$. A prova a $10^{\circ} \mathrm{C}$ apresentou valores de assimetria dentro dos padrões de normalidade em $52,5 \%$ dos casos e valores anormais de assimetria em $16,4 \%$ $(p=0,012)$, confirmou hiporreflexia unilateral em $11,5 \%$ e identificou anormalidades não evidenciadas a 42 e $18^{\circ} \mathrm{C} \mathrm{em} 8,2 \%$. Conclusão: a estimulação gelada com ar a $10^{\circ} \mathrm{C}$ possibilitou retirar a influência do nistagmo espontâneo de olhos fechados sobre o nistagmo pós-calórico em pacientes com vestibulopatias periféricas crônicas.

DESCRITORES: Eletronistagmografia; Nistagmo; Vertigem

(1) Fonoaudióloga; Mestre em Ciências pelo Programa de Pós-graduação em Distúrbios da Comunicação Humana: Campo Fonoaudiológico pela Universidade Federal de São Paulo.

(2) Fonoaudióloga; Professora Adjunta Substituta da Disciplina de Distúrbios da Audição do Departamento de Fonoaudiologia da Universidade Federal de São Paulo; Doutora em Ciências pela Universidade Federal de São Paulo.

(3) Médico; Professor Adjunto da Disciplina de Otologia e Otoneurologia Departamento de Otorrinolaringologia e Cirurgia de Cabeça e Pescoço da Universidade Federal de São Paulo; Professor Colaborador do Programa de PósGraduação em Ciências da Reabilitação Neuro-Motora da Uniban; Doutor em Medicina pela Universidade Federal de São Paulo.

(4) Médico; Professor Titular de Otorrinolaringologia da Universidade Federal de São Paulo; Professor Titular da Universidade Federal de São Paulo; Coordenador do Programa de Mestrado em Reabilitação Vestibular e Inclusão Social da Uniban.

(5) Fonoaudióloga; Professor Associado da Disciplina de Otologia e Otoneurologia Departamento de Otorrinolaringologia e Cirurgia de Cabeça e Pescoço da Universidade Federal de São Paulo; Livre-Docente em Otoneurologia pela Universidade Federal de São Paulo.

\section{INTRODUÇÃO}

A presença, direção, intensidade e morfologia do nistagmo espontâneo auxiliam na definição do topodiagnóstico de uma lesão vestibular periférica ou central ${ }^{1}$. A prova calórica avalia cada labirinto separadamente e permite reconhecer o lado acometido e caracterizar a intensidade da lesão vestibular ${ }^{2}$. O nistagmo espontâneo e o nistagmo pré-calórico ${ }^{3,4}$, identificado na posição em que a prova calórica é realizada, provocam uma preponderância direcional para o mesmo lado ${ }^{5}$, com intensidade inversamente proporcional à soma dos índices das respostas às estimulações quentes e frias ${ }^{6}$, ou podem ocorrer respostas normais, que impossibilitam identificar o labirinto comprometido ${ }^{7}$; o nistagmo espontâneo seria somado à resposta calórica de mesma direção e subtraído da resposta na direção oposta ${ }^{8}$.

A avaliação de predomínio labiríntico e de preponderância direcional às fórmulas de Jongkees, Phillipszoon ${ }^{9}$ é prejudicada ${ }^{1}$ e a influência do 
nistagmo espontâneo sobre o nistagmo pós-calórico não costuma ser constante ${ }^{10}$. A associação de preponderância direcional com hiporreflexia unilateral ocorre principalmente em pacientes com vestibulopatia periférica aguda; com a compensação vestibular, a preponderância direcional diminui progressivamente e a hiporreflexia persiste ${ }^{11}$. Uma prova calórica mais intensa pode ser empregada em pacientes com ausência de respostas ou com nistagmo espontâneo ${ }^{11}$; em pacientes com vestibulopatias crônicas que apresentaram preponderância direcional à prova a 42 e $18^{\circ} \mathrm{C}$ ocasionada por nistagmo espontâneo ou pré-calórico, a prova calórica gelada com ar a $10^{\circ} \mathrm{C}$ retirou a influência destes fenômenos oculares, evidenciando nistagmo póscalórico de intensidade similar nos dois ouvidos ${ }^{12}$.

A freqüente observação da interferência do nistagmo espontâneo de olhos fechados sobre o nistagmo pós-calórico de pacientes com vestibulopatias periféricas crônicas e a escassez de informações pertinentes e recentes na literatura motivaram o interesse em procurar interpretar os resultados da prova calórica com a adição de estimulação gelada com ar a $10^{\circ} \mathrm{C}$ nestes pacientes.

O objetivo deste trabalho é analisar o efeito da estimulação gelada com ar a $10^{\circ} \mathrm{C}$ sobre o nistagmo pós-calórico em pacientes com vestibulopatias periféricas crônicas que apresentam nistagmo espontâneo com olhos fechados.

\section{MÉTODOS}

Para a seleção da casuística foram analisados 1567 exames de função vestibular de pacientes com hipótese diagnóstica de vestibulopatia periférica crônica, estabelecida pelo otorrinolaringologista, em um estudo retrospectivo descritivo. Os pacientes foram atendidos na Disciplina de Otologia e Otoneurologia da Universidade Federal de São Paulo - Escola Paulista de Medicina (UNIFESP EPM) de 2001 a 2006; 674 eram do sexo masculino e 893 do feminino, média de idade 54,8 anos. De 316 pacientes $(20,2 \%)$ que apresentavam nistagmo espontâneo de olhos fechados, foram incluídos 61 exames de pacientes que realizaram a prova calórica com ar a 42,18 e $10^{\circ} \mathrm{C}$; 42 pacientes eram do sexo feminino e 19 do masculino, com média de idade 55,8 anos. Foram excluídos 255 exames de pacientes que não realizaram todas as estimulações ou com artefatos nos traçados que dificultavam a adequada avaliação das respostas nistágmicas.

Os pacientes foram submetidos à anamnese e avaliação audiológica, composta por audiometria tonal liminar, audiometria vocal, medidas de imitância acústica e pesquisa dos reflexos acústicos ${ }^{13}$.
Os pacientes, previamente à avaliação funcional do sistema vestibular, foram orientados a não consumir chocolates, bebidas alcoólicas ou cafeínadas, evitar fumar e usar medicamentos como analgésicos, antivertiginosos e calmantes três dias antes da avaliação. Foram orientados também a permanecerem em jejum por um período de quatro horas antecedentes ao exame e não fazerem uso de maquiagem, cremes faciais ou lentes de contato no dia da avaliação.

Os participantes desta pesquisa foram submetidos a testes que avaliam a função vestibular por meio de vectonistagmografia digital (Neurograff Eletromedicina Ind. e Com. Ltda - EPT) e otocalorímetro a ar (Neurograff Eletromedicina Ind. e Com. Ltda - EPT - Brasil), composta pela pesquisa de nistagmo de posicionamento e posicional, calibração dos movimentos oculares, nistagmo espontâneo e semi-espontâneo, movimentos sacádicos, rastreio pendular, nistagmo optocinético, prova rotatória pendular decrescente e prova calórica com ar a 42,18 e $10^{\circ} \mathrm{C}{ }^{1}$. Os exames foram realizados, sob supervisão, por fonoaudiólogas do Setor de Vestibulometria da Disciplina de Otologia e Otoneurologia da UNIFESP - EPM; os traçados foram avaliados por três fonoaudiólogas e dois médicos, autores desta investigação.

Os indivíduos foram submetidos à limpeza da pele e colocação de três eletrodos de cloreto de prata ativos e um eletrodo terra. Os eletrodos foram dispostos no canto externo peri-orbitário direito, no canto externo peri-orbitário esquerdo e na linha média frontal, utilizando a disposição triangular de derivações que permite identificar a direção do nistagmo e aferir a velocidade angular de sua componente lenta em três canais de registro.

A calibração dos movimentos oculares foi realizada antes da pesquisa do nistagmo espontâneo e da prova calórica, para que os exames fossem realizados em igualdade de condições.

O nistagmo espontâneo foi pesquisado no olhar frontal com os olhos abertos e depois fechados. $O$ nistagmo semi-espontâneo foi investigado no desvio do olhar para a direita, para a esquerda, para cima e para baixo, sem ultrapassar $30^{\circ}$ de desvio da linha média. Na presença destes fenômenos, foram medidas as velocidades das componentes lentas.

Na prova calórica o paciente foi mantido na posição supina com a cabeça elevada $30^{\circ}$. A estimulação térmica foi realizada estimulando cada ouvido separadamente com ar a 42,18 e $10^{\circ} \mathrm{C}$, durante 80 segundos, respeitando um intervalo de três minutos entre uma estimulação e outra. O paciente foi mantido na posição supina com a cabeça elevada $30^{\circ}$ e realizou atividade mental para evitar inibição cortical da resposta calórica. Vertigem, direção e 
velocidade da componente lenta do nistagmo póscalórico foram analisadas com os olhos fechados e com os olhos abertos, para avaliar o efeito inibidor à fixação visual. A presença de nistagmo espontâneo de olhos fechados e/ou pré-calórico e a sua influência nos resultados da prova foram investigadas.

Foram calculados automaticamente os valores de predomínio labiríntico e de preponderância direcional pela fórmula de Jongkees, Philipszoon ${ }^{9}$. $\mathrm{Na}$ prova calórica com ar a 18 e $42^{\circ} \mathrm{C}$, valores de velocidade da componente lenta entre dois e 19 graus por segundo, predomínio labiríntico até $33,0 \%$ e de preponderância direcional até $22,0 \%$ foram considerados dentro do limite da normalidade ${ }^{14}$. Na prova calórica com ar a $10^{\circ} \mathrm{C}$, valores de velocidade da componente lenta acima de dez graus por segundo e assimetria das respostas até $30,0 \%$ foram considerados normais ${ }^{15}$.

A hiporreflexia e a preponderância direcional foram calculadas com base nas fórmulas elaboradas por Jongkees, Phillipszoon ${ }^{9}$, para os valores da velocidade da componente lenta do nistagmo pós-calórico nas quatro estimulações. No cálculo percentual das assimetrias, os valores da velocidade dos batimentos para a direita são assinalados como número negativo e os valores da velocidade dos batimentos para a esquerda são assinalados como número positivo, sendo utilizados diante da presença de nistagmo espontâneo ${ }^{16}$. Desta forma, uma resposta com direção diferente da esperada, por influência do nistagmo espontâneo, é considerada para efeito de cálculo.

Hiporreflexia:

$$
\frac{\left(18^{\circ} D-42^{\circ} D\right)-\left(42^{\circ} E-18^{\circ} E\right)}{18^{\circ} D+42^{\circ} E-18^{\circ} E-42^{\circ} D} \times 100
$$

Preponderância direcional:

$$
\frac{\left(-18^{\circ} E-42^{\circ} D\right)-\left(18^{\circ} D+42^{\circ} E\right)}{18^{\circ} D+42^{\circ} E-18^{\circ} E-42^{\circ} D} \times 100
$$

Assimetria na prova de $10^{\circ} \mathrm{C}$ :

$$
\frac{-10^{\circ} \mathrm{E}-10^{\circ} \mathrm{D}}{-10^{\circ} \mathrm{E}+10^{\circ} \mathrm{D}} \times 100
$$

em que:

$18^{\circ} \mathrm{D}$ : valor da velocidade da componente lenta a $18^{\circ} \mathrm{C}$ no ouvido direito;

$42^{\circ} \mathrm{E}$ : valor da velocidade da componente lenta a $42^{\circ} \mathrm{C}$ no ouvido esquerdo; $18^{\circ} \mathrm{E}$ : valor da velocidade da componente lenta a $18^{\circ} \mathrm{C}$ no ouvido esquerdo;

$42^{\circ} \mathrm{D}$ : valor da velocidade da componente lenta a $42^{\circ} \mathrm{C}$ no ouvido direito;

$10^{\circ} \mathrm{D}$ : valor da velocidade da componente lenta a $10^{\circ} \mathrm{C}$ no ouvido direito;

$10^{\circ} \mathrm{E}$ : valor da velocidade da componente lenta a $10^{\circ} \mathrm{C}$ no ouvido esquerdo.

Os valores limites das fórmulas de preponderância direcional e predomínio labiríntico a 42 e $18^{\circ} \mathrm{C}$ e de assimetria a $10^{\circ} \mathrm{C}$ foram utilizados para avaliar a influência do nistagmo espontâneo de olhos fechados sobre o nistagmo pós-calórico.

O exame otoneurológico possibilitou a caracterização da disfunção do sistema vestibular nos pacientes deste estudo.

Este estudo foi aprovado pelo Comitê de Ética da UNIFESP - EPM sob número de protocolo 01019/05.

Coletada a amostra, os resultados da prova calórica foram submetidos a um estudo estatístico por meio de técnicas estatísticas descritas a seguir, aplicadas com nível de significância de $5 \%(\alpha=0,05)$. $\mathrm{Na}$ análise de associações entre as freqüências de uma amostra com duas categorias utilizou-se o teste Qui-Quadrado de Aderência, seguido pela correção de Yates. Para verificar associação entre as freqüências de uma amostra com três categorias foi empregado o teste Qui-Quadrado de Aderência.

\section{RESULTADOS}

Foram avaliados a direção e valores em graus por segundo da velocidade da componente lenta do nistagmo espontâneo de olhos fechados, a presença ou ausência de nistagmo pré-calórico, valores em graus por segundo da velocidade da componente lenta do nistagmo pós-calórico a 42 e $18^{\circ} \mathrm{C}$, valores percentuais de preponderância direcional e de predomínio labiríntico a 42 e $18^{\circ} \mathrm{C}$, valores em graus por segundo da velocidade da componente lenta do nistagmo pós-calórico a $10^{\circ} \mathrm{C}$ e valores percentuais da assimetria a $10^{\circ} \mathrm{C}$.

O nistagmo espontâneo de olhos fechados foi horizontal para a direita em 30 casos $(49,2 \%)$, oblíquo para a direita e para baixo em seis casos $(9,8 \%)$, horizontal para a esquerda em 19 casos $(31,1 \%)$, oblíquo para a esquerda e para cima em cinco casos $(8,2 \%)$ e oblíquo para a esquerda e para baixo em um caso (1,6\%); 34 casos $(55,7 \%)$ também apresentaram nistagmo pré-calórico na mesma direção.

$\mathrm{Na}$ prova calórica a 42 e $18^{\circ} \mathrm{C}$, nistagmo na mesma direção do nistagmo espontâneo e na direção contrária à esperada foi verificado em 
17 casos (27,9\%). Preponderância direcional anormal ocorreu em 18 casos $(29,5 \%)$, predomínio labiríntico anormal em sete $(11,5 \%)$ e preponderância direcional e predomínio labiríntico anormais em 17 (27,9\%). Não foram encontrados valores anormais de preponderância direcional e/ ou de predomínio labiríntico em 19 casos (31,1\%). O número de casos que apresentaram valores anormais de preponderância direcional e/ou de predomínio labiríntico foi significativamente maior do que o número de casos com valores normais $(p=0,049)$. Esse comentário faz parte da discussão e não de resultados.

Na prova calórica a $10^{\circ} \mathrm{C}$, nistagmo na mesma direção do nistagmo espontâneo e na direção contrária à esperada foi observado em dois casos $(3,3 \%)$. Não houve diferença significante entre o número de casos (24 ou $39,3 \%$ ) com valores anormais de assimetria e o número de casos ( 37 casos ou $60,7 \%$ ) com valores de assimetria dentro do padrão de normalidade $(p=0,124)$. Hiporreflexia unilateral foi confirmada em sete casos $(11,5 \%)$.

Nos 42 casos $(69,8 \%)$ que apresentaram valores anormais de preponderância direcional e/ou de predomínio labiríntico a 42 e $18^{\circ} \mathrm{C}$, a prova a $10^{\circ} \mathrm{C}$ apresentou um número significativamente maior de casos (32 ou 52,5\%) com valores de assimetria dentro do padrão de normalidade quando comparados ao número de casos (10 ou 16,4\%) com valores anormais de assimetria $(p=0,012)$.

Dos 19 casos $(31,1 \%)$ com valores normais de preponderância direcional e/ou de predomínio labiríntico a 42 e $18^{\circ} \mathrm{C}$, cinco casos $(8,2 \%)$ evidenciaram valores anormais de assimetria à prova a $10^{\circ} \mathrm{C}$.

\section{DISCUSSÃO}

Nessa casuística, verificou-se a presença de nistagmo espontâneo horizontal para a direita, horizontal para a esquerda, oblíquo para a direita e para baixo, oblíquo para a esquerda e para cima ou oblíquo para a esquerda e para baixo, em ordem decrescente de prevalência. A ocorrência de nistagmo pré-calórico foi observada na mesma direção do nistagmo espontâneo em mais da metade da casuística. O nistagmo pré-calórico é considerado como o próprio nistagmo espontâneo presente na posição corporal em que a prova calórica é realizada ${ }^{3,4}$.

Na prova calórica a 42 e $18^{\circ} \mathrm{C}$, ocorreram preponderância direcional e/ou de predomínio labiríntico dentro do padrão de normalidade, preponderância direcional anormal, preponderância direcional e predomínio labiríntico anormais ou predomínio labiríntico anormal, em ordem decrescente de prevalência; o número de casos com anormalidades do nistagmo pós-calórico prevaleceu sobre o número de casos sem anormalidades. O nistagmo espontâneo modifica a intensidade das respostas calóricas e produz uma preponderância direcional para o mesmo lado ${ }^{6}$, mas a influência quantitativa do nistagmo espontâneo sobre o nistagmo pós-calórico não costuma ser constante ${ }^{10}$.

A prova calórica mais intensa permite a investigação da função vestibular de pacientes com nistagmo espontâneo, com ausência de respostas ou quando há dúvidas quanto à presença de respostas pela interferência de um nistagmo espontâneo, confirmando ou não uma arreflexia ou hiporreflexia severa ${ }^{11}$.

Nas provas a 42 e $18^{\circ} \mathrm{C}$ e a $10^{\circ} \mathrm{C}$, nistagmo póscalórico dentro dos padrões da normalidade, sem influência do nistagmo espontâneo, foi encontrado em parte da casuística, o que é concordante com informações da literatura ${ }^{6}$.

Após uma ou mais estimulações térmicas da prova a 42 e $18^{\circ} \mathrm{C}$ ou a $10^{\circ} \mathrm{C}$, observamos nistagmo na mesma direção do nistagmo espontâneo e direção contrária à esperada em alguns casos, em que a estimulação térmica não foi capaz de evidenciar um nistagmo provocado.

Na prova calórica a $10^{\circ} \mathrm{C}$, apesar da maioria dos casos ter apresentado valores normais de assimetria, não houve diferença significante à comparação com o número de casos com valores anormais. Em pacientes com vestibulopatias periféricas crônicas, a prova calórica gelada com ar a $10^{\circ} \mathrm{C}$ retirou a influência de nistagmo espontâneo e pré-calórico, evidenciando nistagmo pós-calórico de intensidade similar nos dois ouvidos ${ }^{12}$.

No grupo de pacientes com valores anormais de preponderância direcional e/ou de predomínio labiríntico a 42 e $18^{\circ} \mathrm{C}$, foi possível verificar que a prova a $10^{\circ} \mathrm{C}$ apresentou um número maior de casos com valores de assimetria dentro do padrão de normalidade do que de casos com valores anormais de assimetria, indicando a retirada da interferência do nistagmo espontâneo na maioria dos casos e também confirmou a presença de hiporreflexia unilateral em alguns casos, corroborando informações da literatura sobre a utilidade de uma estimulação calórica mais intensa para a identificação das síndromes vestibulares deficitárias ${ }^{11}$.

A prova calórica a $10^{\circ} \mathrm{C}$ identificou anormalidades não evidenciadas a 42 e $18^{\circ} \mathrm{C}$ em alguns casos. Não foram encontradas citações na literatura sobre este achado.

A possibilidade de retirada da influência do nistagmo espontâneo de olhos fechados, de confirmação de hiporreflexia unilateral e de identificação de anormalidades não evidenciadas a 42 e $18^{\circ} \mathrm{C}$ 
demonstra a utilidade da realização da estimulação a $10^{\circ} \mathrm{C}$ com ar, auxiliando na interpretação dos resultados da prova calórica e contribuindo para o estabelecimento da hipótese diagnóstica, orientação terapêutica e prognóstica em pacientes com disfunção vestibular periférica.

\section{CONCLUSÃO}

A estimulação gelada com ar a $10^{\circ} \mathrm{C}$ possibilitou retirar a influência do nistagmo espontâneo de olhos fechados sobre o nistagmo pós-calórico em pacientes com vestibulopatias periféricas crônicas.

\begin{abstract}
Purpose: to analyze the effect of air caloric stimulation at $10^{\circ} \mathrm{C}$ on the post-caloric responses in patients with chronic peripheral dysfunction who presented spontaneous nystagmus with the eyes closed. Methods: 61 patients with spontaneous nystagmus with closed eyes were submitted to air caloric stimulation under the following temperatures: 42,18 and $10^{\circ} \mathrm{C}$. Results: in 42 patients $(69.8 \%)$, abnormal values of directional preponderance and / or unilateral hypoexcitability were observed following the $42^{\circ} \mathrm{C}$ and $18^{\circ} \mathrm{C}$ stimulations. For the $10^{\circ} \mathrm{C}$ stimulations an asymmetry within normal limits was seen in $52.5 \%$ of the patients, while abnormal values were seen in $16.4 \%(p=0.012)$. Unilateral hypoexcitability was confirmed in $11.5 \%$ of the cases. In $8.2 \%$ of the patients there were abnormal findings not evidenced under $42^{\circ} \mathrm{C}$ and $18^{\circ} \mathrm{C}$ stimulations. Conclusion: in patients with chronic peripheral labyrinthine disorders who show spontaneous nystagmus with closed eyes, the $10^{\circ} \mathrm{C}$ caloric test makes it possible to remove the influence of the spontaneous nystagmus on the post-caloric responses.
\end{abstract}

KEYWORDS: Electronystagmography; Nystagmus; Vertigo

\section{REFERÊNCIAS}

1. Mangabeira Albernaz PL, Ganança MM, Caovilla $\mathrm{HH}$. Critérios em vestibulometria. Acta AWHO. 1982; (Supl 2):1-63.

2. Baloh RW, Furman JM. Modern vestibular function testing. West J Med. 1989; 150(1):59-67.

3. Dayal VS, Tarantino L, Farkashidy J, Paradisgarten A. Spontaneous and positional nystagmus: a reassessment of clinical significance. Laryngoscope. 1974; 84(11):2033-44.

4. Toupet M, Moreau JL, Frachet B, Pialoux P. Le nystagmus pseudo-calorique. Ann Oto-laryng. 1981; 98:353-8.

5. Fitzgerald G, Hallpike CS. Studies in human vestibular function. I: observations on the directional preponderance ("nystagmusbereitschaft") of caloric nystagmus resulting from cerebral lesions. Brain. 1942; 62(part 2):115-37.

6. Toupet M. Comment le nystagmus spontané modifie le nystagmus provoqué par la stimulation vestibulaire calorique, chez 34 patients atteints de névrite vestibulaire. Ann Oto-laryng. 1982; 99:453-64.

7. Downie DB, Simmons FB. Spontaneous nystagmus direction does not indicate laterality. Arch Otolaryngol. 1975; 101(6):358-60.
8. Baloh RW, Sills AW, Honrubia V. Caloric testing. 3. Patients with peripheral and central vestibular lesions. Ann Otol Rhinol Laryngol Suppl. 1977; 86(5 Pt 3 Suppl 43):24-30.

9. Jongkees LB, Philipszoon AJ. Electronystagmography. Acta Otolaryngol Suppl. 1964; 189:Suppl 189:1+.

10. Ganança MM, Mangabeira Albernaz PL, Caovilla HH, Ito YI, Novo NF, Juliano Y et al. Como diagnosticar e tratar a vertigem. Rev Bras Med. 1987; 44(6):143-56.

11. Jacobson GP, Newman CW, Kartush JM. Handbook of balance function testing. St. Louis: Mosby; 1993. 439p.

12. Frazza M, Cabete CF, Gushikem $P$, Sanchez ML, Freitas Ganança C, Câmera JLS et al. Exemplos da capacidade de informação do laboratório otoneurológico. In: Ganança MM, Munhoz MSL, Caovilla HH, Silva MLG. Otoneurologia ilustrada. São Paulo: Atheneu; 2005. p.13-140.

13. Munhoz MSL, Caovilla HH, Silva MLG, Ganança MM. Audiologia Clínica. São Paulo: Atheneu; 2000. 284p.

14. Freitas Ganança C, Souza JAC, Segantin LA, Caovilla HH, Ganança MM. Limites normais dos parâmetros de avaliação à vectoelectronistagmografia digital Neurograff. Acta AWHO. 2000; 19(2):105. 
15. Caovilla HH, Ganança MM, Mangabeira Albernaz PL. Computer analysis in vectornystagmography. IX Scientific Meeting of the Neurootologial and Equilibriometric Society, Bad Kissingen (Germany), março de 1982.
16. Shepard NT, Telian SA. Practical management of the balance disorder patient. San Diego: Singular; 1996. 221p.

RECEBIDO EM: 10/10/2007

ACEITO EM: 16/07/2008

Endereço para correspondência:

Rua Plínio Colas, 388 ap. 103

São Paulo - SP

CEP: 02435-030

Tel: (11) 6238-7975 / (11) 8196-7972

Fax: (11) 6950-1229

E-mail: flavia_silveira@terra.com.br 